\title{
Structural and electronic properties of atomically thin germanium selenide polymorphs
}

\author{
Shengli Zhang ${ }^{1}$, Shangguo Liu ${ }^{2}$, Shiping Huang ${ }^{2}$, Bo Cai ${ }^{1}$, Meiqiu Xie ${ }^{1}$, Lihua Qu ${ }^{1}$, Yousheng Zou ${ }^{1}$, \\ Ziyu $\mathrm{Hu}^{3 *}$, Xuechao $\mathrm{Yu}^{4}$ and Haibo Zeng ${ }^{1 *}$
}

\begin{abstract}
Using comprehensive density functional theory calculations, we systematically investigate the structure, stability, and electronic properties of five polymorphs of GeSe monolayer, and highlight the differences in their structural and electronic properties. Our calculations show that the five free-standing polymorphs of $\mathrm{GeSe}$ are stable semiconductors. $\beta$-GeSe, $\gamma$-GeSe, $\delta$-GeSe, and $\varepsilon-G e S e$ are indirect gap semiconductors, whereas $\alpha-G e S e$ is a direct gap semiconductor. We calculated Raman spectra and scanning tunneling microscopy images for the five polymorphs. Our results show that the $\beta$-GeSe monolayer is a candidate for water splitting.
\end{abstract}

\section{INTRODUCTION}

Two-dimensional (2D) materials have gained increasing attention since graphene, the monolayer counterpart of graphite, was isolated by mechanical exfoliation [1]. Thereafter, interest expanded to other 2D materials, such as silicene, hexagonal boron nitride, molybdenum disulphide, phosphorene, arsenene and antimonene [2-8]. Among the $2 \mathrm{D}$ materials, chalcogenide nanostructures with layered structures of group IV elements (GeS, GeSe, SnS, SnSe, etc.) have attracted considerable attention because of their exceptional properties and promising applications [9-15]. Bulk Ge-based chalcogenides, such as GeSe, have been extensively studied owing to their unique optical and electronic properties, as well as their high stability, abundance, environmental friendliness, and low toxicity [16-19]. However, a comprehensive study of the stability and properties of single-layered GeSe is still missing.

The polymorphs of $2 \mathrm{D}$ monolayers have received significant attention, such as the polymorphs of $\mathrm{P}, \mathrm{As}$, and $\mathrm{Sb}$ monolayers [20-24]. $\alpha$-SiS has been proposed as an isoelec- tronic counterpart to the layered group V semiconductors [25]. Similarly, GeSe is an isoelectronic counterpart to the group V semiconductors and has the same layered structures. $a-G e S e$ nanosheets have been synthesized by several methods and applied in photodetector devices [11,12]. The semiconducting electronic properties of $\alpha-\mathrm{GeSe}$ have been confirmed by a recent theoretical investigation [26]. However, the stability and electronic properties of the GeSe monolayers in the other polymorphs have not been investigated. A systematic theoretical investigation of their structures and properties will not only enrich our understanding of the property variations of the polymorphs, but it will also facilitate potential applications. Despite some theoretical and experimental studies of $a-G e S e$ being reported, many important questions still remain unknown: what are the stabilities of the GeSe monolayers in the different phases? How do the electronic properties vary with the different phase structures? In this work, we aim to answer these questions.

In this work, by comprehensive density functional theory (DFT) calculations, we report the discovery of the four previously unknown phases of the GeSe monolayer in addition to layered $\alpha$-GeSe: $\beta$-GeSe, $\gamma$-GeSe, $\delta$-GeSe, and $\varepsilon$-GeSe. The five polymorphs of GeSe exhibit versatile energy band gaps, which are very significant for broadband optoelectronic and photonic applications. In particular, $\beta-\mathrm{GeSe}$ is an indirect band-gap semiconductor with a band gap of $3.01 \mathrm{eV}$ calculated using the HSE06 exchange-correlation functional. From the band edge alignment, the conduction band minimum (CBM) and valence band maximum (VBM) energies of $\beta-\mathrm{GeSe}$ are -2.54 and $-5.55 \mathrm{eV}$, respectively, which indicates that it is a promising material

\footnotetext{
${ }^{1}$ Institute of Optoelectronics \& Nanomaterials, Herbert Gleiter Institute of Nanoscience, College of Materials Science and Engineering, Nanjing University of Science and Technology, Nanjing 210094, China

${ }^{2}$ State Key Laboratory of Organic-Inorganic Composites, Beijing University of Chemical Technology, Beijing 100029, China

${ }^{3}$ Beijing Computational Science Research Center, Beijing 100094, China

${ }^{4}$ PTIMUS, Centre for OptoElectronics and Biophotonics, School of Electrical and Electronic Engineering, Nanyang Technological University, Singapore 639798, Singapore

* Corresponding authors (emails: huziyu@csrc.ac.cn (Hu Z); zeng.haibo@njust.edu.cn (Zeng H))
} 
for efficient water splitting.

\section{COMPUTATIONAL METHODS}

All of the calculations, including geometry relaxation, energy calculations, and phonon calculations, were performed using the Cambridge Sequential Total Energy Package (CASTEP) with a norm-conserving pseudo-potential [27]. The exchange-correlation functional was treated using the generalized gradient approximation with the Perdew-Wang exchange-correlation functional (PW91) [28,29]. Periodic boundary conditions were used to simulate an infinite GeSe monolayer. A sufficiently large vacuum region of $20 \AA$ was used to separate the $2 \mathrm{D}$ structures to prevent any interaction between neighboring layers along the $z$ axis. The structure was optimized by the Broyden-Fletcher-Goldfarb-Shanno (BFGS) method [30]. The basis set for the valence electronic states was set to $4 \mathrm{~s}^{2} 4 \mathrm{p}^{2}$ for Ge and $4 \mathrm{~s}^{2} 4 \mathrm{p}^{4}$ for Se. All of the structure models were fully relaxed until the forces were less than 0.01 $\mathrm{eV} \AA^{-1}$. The self-consistent convergence was set to $5 \times 10^{-6}$ $\mathrm{eV}$ per atom. A plane wave basis set with a cutoff energy of $440 \mathrm{eV}$ was used. For Brillouin-zone sampling, we used the Monkhorst-Pack mesh with $13 \times 13 \times 3 k$-points [31]. To overcome the problem of band-gap underestimation in the semi-local exchange-correlation functional, the band structures were evaluated using both the PW91 functional and the screened hybrid HSE06 functional, which typically gave a better description of band gaps in semiconductors [32]. To explain the difference of the band edge position in the five GeSe monolayer polymorphs, we first calculated the work functions (WFs). And then, by setting the vacuum energy as zero, the position of VBM can be located as the negative value of WF. The VBM and CBM of the five GeSe monolayer polymorphs with respect to the vacuum level can be calculated.

\section{RESULTS AND DISCUSSION}

The optimized geometric structures of the five different GeSe monolayers are shown in Fig. 1. The results of the optimized geometric structures, such as lattice parameters and thickness of the GeSe monolayer, are summarized in Table 1. The five polymorphs of the GeSe monolayer form honeycomb structures with two sublayers, where each atomic species is covalently bonded to three neighboring atoms of the other atomic species. The ridge structures of $\alpha$-GeSe, $\gamma$-GeSe, $\delta$-GeSe, and $\varepsilon$-GeSe are anisotropic, while $\beta-\mathrm{GeSe}$ has an isotropic structure. The equilibrium configuration of black-phosphorus-like $\alpha$-GeSe has a puckered structure that forms zigzag chains along the $y$ axis, where the individual layers are weakly coupled to the adjacent layers by van der Waals forces [19]. For the orthorhombic a-GeSe monolayer structure, four atoms in the unit cell are
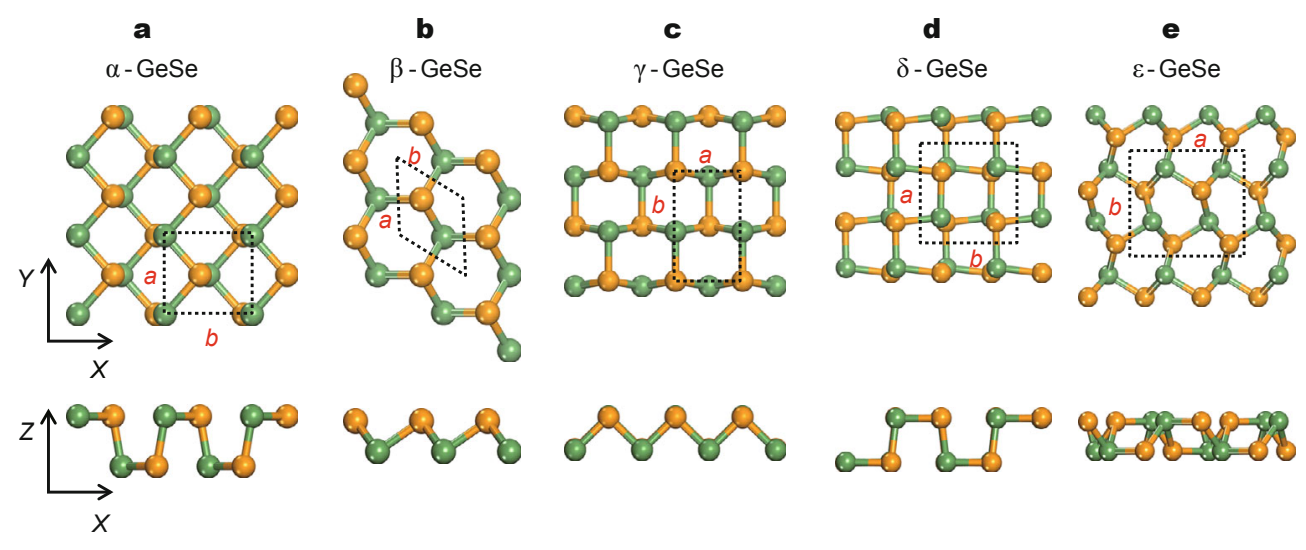

Figure 1 Top (upper) and side (lower) views of the optimized geometric structures of (a) $\alpha$-GeSe, (b) $\beta$-GeSe, (c) $\gamma$-GeSe, (d) $\delta$-GeSe, and (e) $\varepsilon$-GeSe monolayers. Green balls denote Ge atoms and yellow balls denote Se atoms, where $a$ and $b$ represent the lattice parameters. The Wigner-Seitz cells are indicated by dashed lines.

Table 1 Structural properties of the GeSe polymorphs $s^{a}$

\begin{tabular}{ccccccc}
\hline & $\alpha-G e S e$ & $\alpha-G e S e$ & $\beta-G e S e$ & $\gamma$-GeSe & $\delta$-GeSe & $\varepsilon$-GeSe \\
\hline$a(\AA)$ & $3.99^{\mathrm{b}}$ & 3.94 & 3.63 & 3.63 & 5.81 & 6.80 \\
$b(\AA)$ & $4.26^{\mathrm{b}}$ & 4.24 & 3.63 & 5.83 & 5.77 & 6.34 \\
$h(\AA)$ & $\ldots$ & 2.49 & 1.41 & 1.73 & 2.49 & 1.74 \\
\hline
\end{tabular}

a) $a$ and $b$ are the lattice parameters and $h$ is the thickness of the GeSe monolayer; b) data according to Ref. [33]. 
arranged in a rectangular lattice, and the relaxed lattice parameters of $a=3.94 \AA$ and $b=4.24 \AA$ are in agreement with previous theoretical calculations [33]. The calculated bond lengths and bond angles of the five systems are summarized in Fig. S1 (Supplementary Information). The optimized geometric structure of $\beta-G e S e$ is shown in Fig. 1b. $2 \mathrm{D}$ hexagonal $\beta$-GeSe has symmetric lattice vectors $a=b=$ $3.63 \AA$. It has a buckled surface and maintains $\mathrm{sp}^{3}$ character bonds, similar to what has been predicted for blue phosphorus $(\beta-\mathrm{P})$. Two atoms in the unit cell are arranged in a diamond lattice. The thickness of $\beta-G e S e$ is less than the thicknesses of the other polymorphs. $\gamma$-GeSe, $\delta$-GeSe, and $\varepsilon$-GeSe have rectangular unit cells containing four, eight, and eight atoms, respectively.

To obtain insight into the thermodynamic stability of the five polymorphs of GeSe monolayer, we calculated the binding energies (see Fig. 2a). We define the binding energy as $E_{b}=\left(n E_{\mathrm{Ge}}+n E_{\mathrm{Se}}-E_{(\mathrm{GeSe}) n}\right) / n$ where $E_{\mathrm{Ge}}, E_{\mathrm{Se}}$, and $E_{(\mathrm{GeSe}) n}$ are the total energies of a single $\mathrm{Ge}$ atom, Se atom, and $\mathrm{Ge}_{n} \mathrm{Se}_{n}$ monolayer, respectively. The binding energies of $\alpha$-GeSe, $\beta$-GeSe, $\gamma$-GeSe, $\delta$-GeSe, and $\varepsilon$-GeSe are 3.88, $3.85,3.83,3.87$, and $3.78 \mathrm{eV}$, respectively, which is clear evidence that all five polymorphs of GeSe are strongly covalently bonded networks. Among the five $2 \mathrm{D}$ structures, $a-G e S e$ is the most energetic form in these five polymorphs. The orthorhombic structure of bulk $\alpha-G e S e$ (space group $\left.\operatorname{Pcmn}\left(\mathrm{D}_{2 \mathrm{~h}}^{16}\right)\right)$ is stable under ambient conditions. Therefore, all of the five polymorphs of the GeSe monolayer can be considered to be stable structures because their binding energy differences are less than $0.1 \mathrm{eV}$. As a result, we believe that the $\beta$-GeSe, $\gamma$-GeSe, $\delta$-GeSe, and $\varepsilon$-GeSe can also be formed and realized in experiments.

To further investigate the dynamical stabilities of the single-layer GeSe polymorphs, we then performed phonon dispersion calculations (see Figs $2 \mathrm{~b}-\mathrm{f}$ ) along the high symmetric points in the Brillouin zone (Fig. S2). Phonon calculations can give a criterion for crystal stability, where soft modes indicate structure instability [34]. There are no imaginary frequencies in the five systems, which means that they are highly kinetically stable. From detailed analysis of the phonon spectra, the highest frequencies of the five systems are all above $220 \mathrm{~cm}^{-1}$.

To better understand the electronic properties, we calculated the band structures of the five GeSe polymorphs, as shown in Figs 3a-e. All of the five structures are semiconductors. For $\alpha-\mathrm{GeSe}, \beta-\mathrm{GeSe}, \gamma-\mathrm{GeSe}, \delta$-GeSe, and $\varepsilon$-GeSe, the energy band gaps calculated using the PW91 (HSE06) functional are 1.23 (1.75), 2.32 (3.01), 1.71 (2.34), 1.75 (2.27), and 1.85 (2.47), respectively. Fig. 3a shows the band structures of a-GeSe obtained using the HSE06 hybrid functional (red curve) and PW91 (black curve) functional. The result for the HSE06 hybrid functional shows that a-GeSe is a semiconductor with a quasi-direct band gap. Close inspection of the two conduction band edges near Fermi level reveals a small deviation of less than $0.1 \mathrm{eV}$. The result using PW91 shows that $a-G e S e$ is a direct band gap
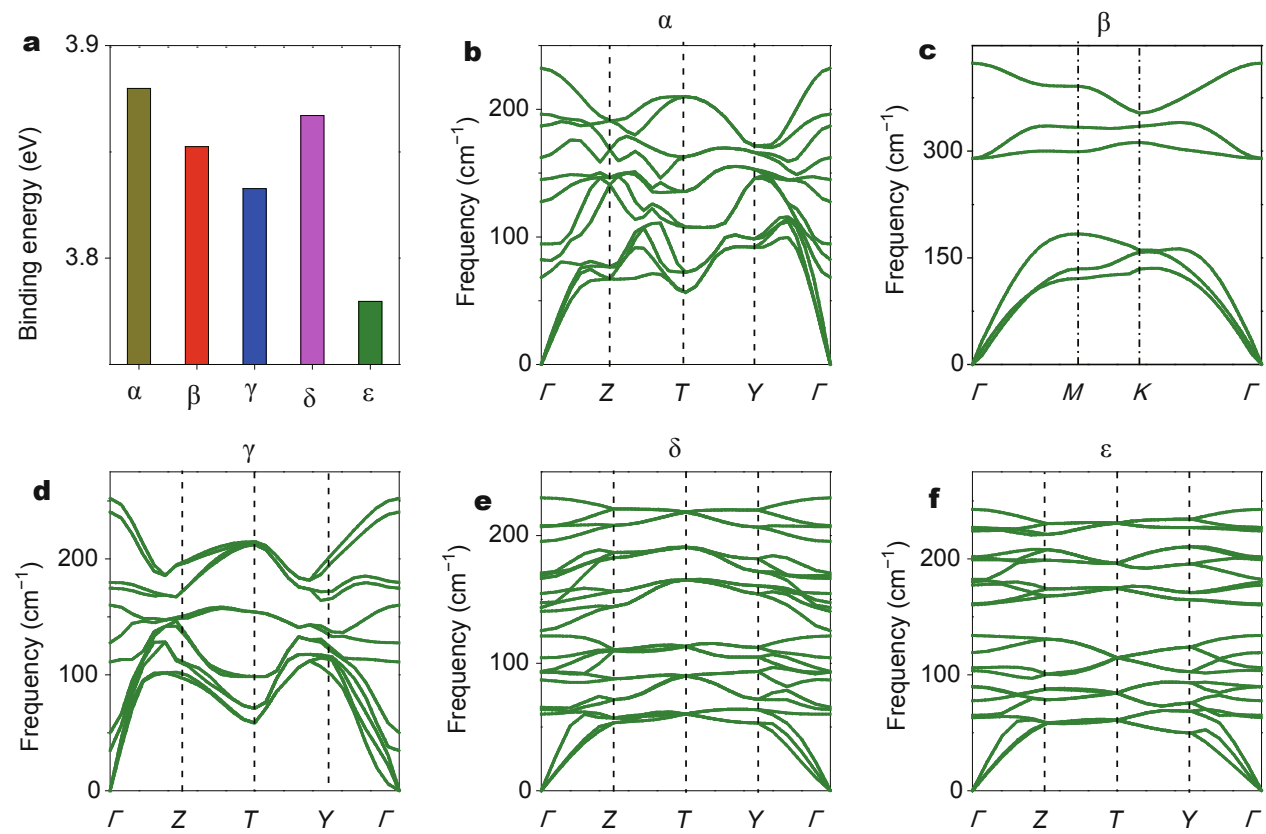

Figure 2 (a) Calculated binding energies and (b)-(f) phonon dispersions of the GeSe polymorphs. (b) $\alpha$-GeSe, (c) $\beta$-GeSe, (d) $\gamma$-GeSe, (e) $\delta$-GeSe, and (f) $\varepsilon$-GeSe. 

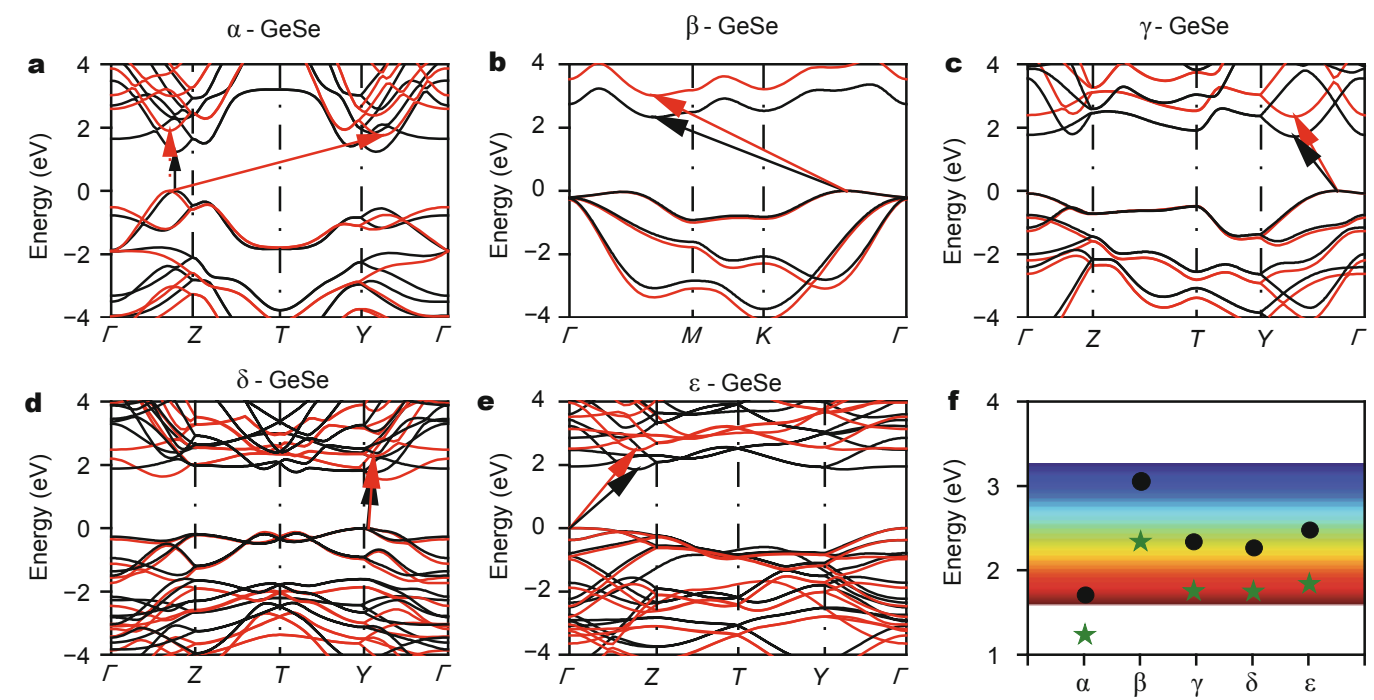

Figure 3 (a)-(e) Electronic band structures of the GeSe monolayer polymorphs, where black lines represent the results with the PW91 functional and red lines are the results with the HSE06 method. The VBM and CBM are highlighted by black and red single arrows, respectively. (f) Bandgaps calculated using the HSE06 (black circles) and PW91 (green stars) functional. The visible spectrum is overlaid to aid visualization of the bandgap.

with the VBM and CBM located in the $\Gamma-Z$ direction. Interestingly, the valence band of $\alpha-\mathrm{GeSe}$ exhibits unexpected symmetry along the $\Gamma-Z$ and $\Gamma-Y$ directions. The main advantage of $\beta-\mathrm{GeSe}$ is its wide fundamental band gap of greater than $3 \mathrm{eV}$ (HSE06), which indicates that it can be used in optoelectronic devices such as blue-light emitting diodes and photodetectors. The band structure of $\beta$-GeSe near the top of the valence band shows significant isotropy comparing the $\Gamma-M$ and $\Gamma-K$ directions. Neither the VBM nor the CBM of $\beta$-GeSe, $\gamma$-GeSe and $\delta$-GeSe lies at the high-symmetry $k$ points in the Brillouin zone. In $\varepsilon-G e S e$, the CBM and VBM occur along the $\Gamma-Z$ direction and at the $Y$ point, respectively. Moreover, as shown in Fig. 3, the differences in energies between the two valence-band edges near Fermi level are very small for $\beta$-GeSe, $\gamma$-GeSe, $\delta$-GeSe, and $\varepsilon$-GeSe $(36,84,129$, and $0 \mathrm{meV}$, respectively) compared with $\alpha-\mathrm{GeSe}(>500 \mathrm{meV})$ using the HSE06 functional. For $\delta$-GeSe, the top of the valence band is very flat, resulting in a heavy hole mass and a large density of states (DOS) in that region.

For the design of devices, it is important to know the intrinsic band offsets between the five GeSe polymorphs. Fig. S3 shows the band edge alignment of the GeSe polymorphs obtained using the HSE06 functional. The CBM and VBM energies of $\beta-\mathrm{GeSe}$ are -2.54 and $-5.55 \mathrm{eV}$, respectively, indicating that a bias potential of $0.12 \mathrm{eV}$ is sufficient for water splitting and compression strains can reduce the required bias potential [35].

To understand the contribution of different orbitals to the electronic states, we calculated the total DOS and partial DOS (PDOS) for the five polymorphs, and the results are summarized in Fig. 4. From detailed analysis of the PDOS of GeSe, the states near Fermi level have contributions from both $\mathrm{s}$ and $\mathrm{p}$ orbitals. However, the contributions from $\mathrm{p}$ orbitals to the total DOS are much greater than those from s orbitals. The fact that $\mathrm{p}$ orbitals are dominant is a common feature of single-layer honeycomb systems, such as silicene and phosphorene [20]. From detailed analysis of the PDOS of $a-G e S e$, the VBM is dominated by the $4 \mathrm{p}$ orbitals of the Se atoms. The CBM is dominated by the $4 \mathrm{p}$ orbitals of $\mathrm{Ge}$ atoms. For the $\beta-\mathrm{GeSe}$ monolayer, the main contribution to the VBM is from Se $4 p$ states and partially from $\mathrm{Ge} 4 \mathrm{p}$ states, while the main contributions to the CBM are from Ge $4 p$ states and partially from Se $4 p$ states.

Raman spectroscopy is a widely used experimental technique to identify the characteristic vibrational modes of materials. It is one of the most commonly used optical techniques for the characterization of 2D materials [36]. Several properties can be determined from analysis of the Raman spectra. For example, the shift in frequency of the Raman peaks has been successfully used to quantify (or identify) the number of layers of layered materials such as $\mathrm{MoS}_{2}$ [37]. In this paper, to provide more information for possible experimental observations, we calculated the Raman spectra for the five GeSe polymorphs, as shown in Fig. 5a. From detailed analysis of the Raman spectra and the corresponding vibrational modes of $\alpha-G e S e$ (see in Fig. $5 \mathrm{~b}$ ), which belongs to the $C_{2 V}$ point group, the Raman ac- 

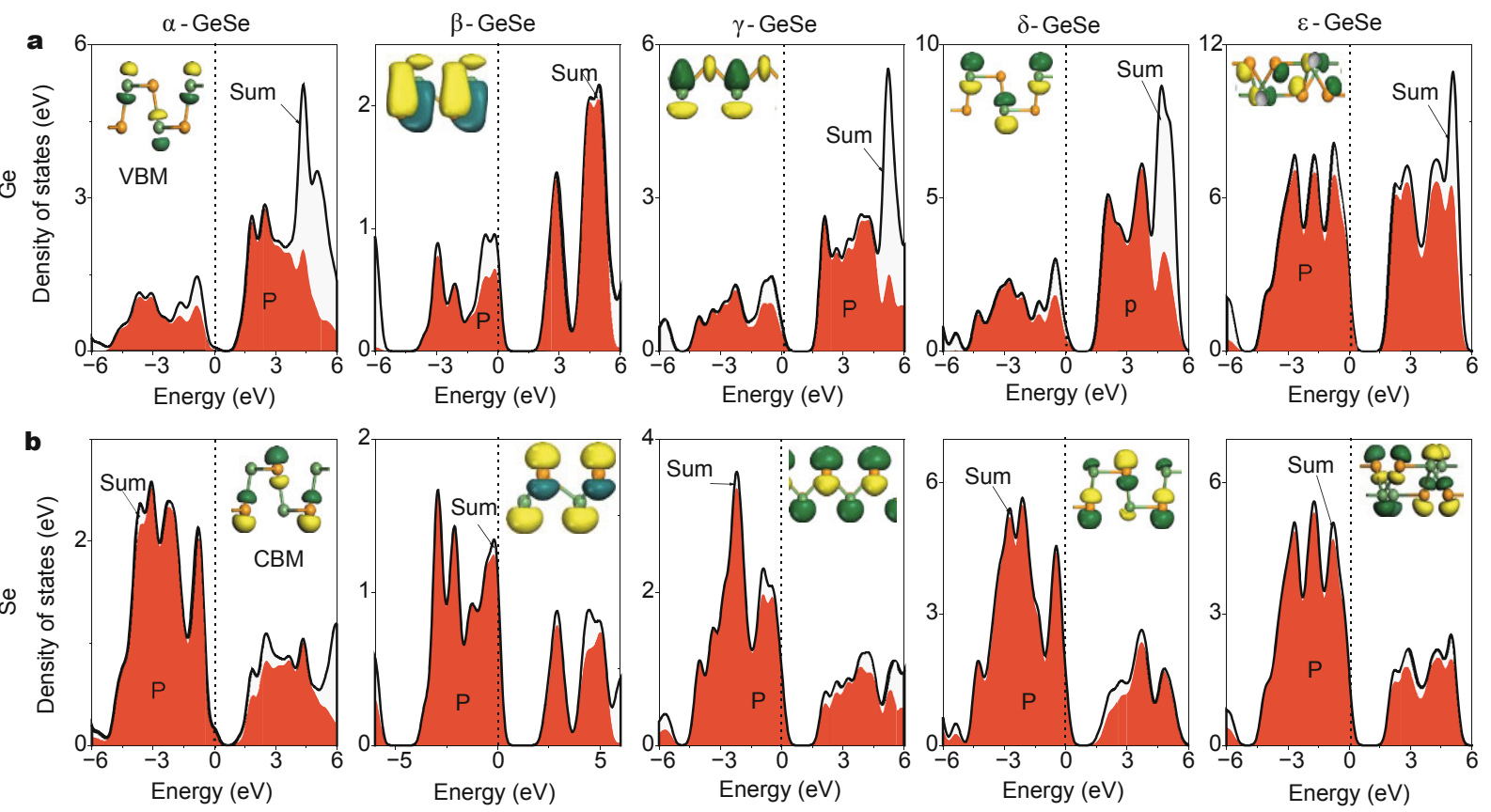

Figure 4 (a) PDOS of Ge with 4p states, (b) PDOS of Se with 4p states in monolayer GeSe polymorphs. The isosurfaces of the partial charge densities for the VBM (a) and CBM (b) of the GeSe monolayer are shown within the PDOS.
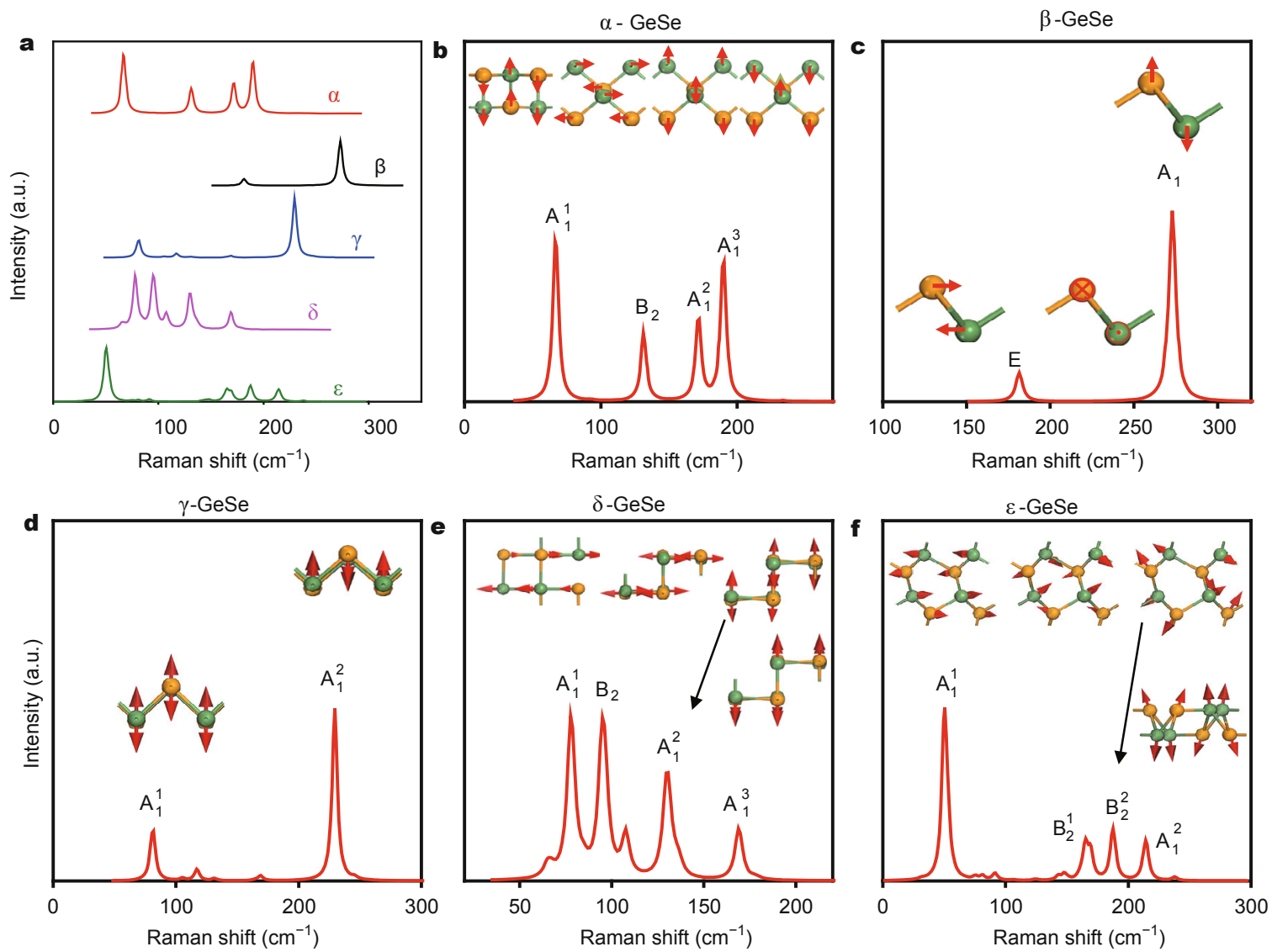

Figure 5 (a) Calculated Raman spectra of the GeSe monolayer polymorphs and (b)-(f) the corresponding vibrational modes. 
tive modes $A_{1}^{1}$ at $67 \mathrm{~cm}^{-1}, B_{2}$ at $131 \mathrm{~cm}^{-1}, A_{1}^{2}$ at $172 \mathrm{~cm}^{-1}$, and $\mathrm{A}_{1}^{3}$ at $190 \mathrm{~cm}^{-1}$ exhibit prominent Raman scattering. The $A_{1}^{1}$ mode is the dominant Raman peak for $a-G e S e$, for which atoms of the same species belonging to the two sublayers vibrate opposite to each other. $B_{2}, A_{1}^{2}$, and $A_{1}^{3}$ are in-plane vibration modes. For $B_{2}$ and $A_{1}^{3}$, atoms belonging to the same species vibrate along opposite directions. Our measurements reveal that the most prominent Raman peaks for $\beta$-GeSe, $\gamma$-GeSe, $\delta$-GeSe, and $\varepsilon$-GeSe are located at 272.9, $229.4,77.6$, and $50.6 \mathrm{~cm}^{-1}$, respectively. In addition, scanning tunneling microscopy images of the five polymorphs were simulated at +2.0 and $-2.0 \mathrm{~V}$ bias to recognize the polymorphs in future experiments, as shown in Fig. S4.

\section{CONCLUSIONS}

In summary, we have theoretically investigated the structural and electronic properties of GeSe monolayer polymorphs using DFT calculations. The thermodynamic stability is given by the binding energy, and the dynamical stability is determined by the phonon spectrum. To further assist in device design, we have calculated the band gaps of the polymorphs. Unlike graphene, which needs to be functionalized to open a band gap, GeSe possesses an intrinsic band gap and can be considered as a new type of $2 \mathrm{D}$ material for applications in optoelectronics and electronic devices.

\section{Received 3 December 2015; accepted 21 December 2015;} published online 25 December 2015

1 Novoselov KS, Geim AK, Morozov S, et al. Electric field effect in atomically thin carbon films. Science, 2004, 306: 666-669

2 Jose D, Datta A. Structures and chemical properties of silicene: unlike graphene. Acc Chem Res, 2013, 47: 593-602

3 Zhang SL, Xie MQ, Li FY, et al. Semiconducting group 15 monolayers: a broad range of band gaps and high carrier mobilities. Angew Chem Int Ed, 2015, DOI: 10.1002/anie.201507568

4 Liu H, Neal AT, Zhu Z, et al. Phosphorene: an unexplored 2D semiconductor with a high hole mobility. ACS nano, 2014, 8: 4033-4041

5 Mak KF, Lee C, Hone J, et al. Atomically thin $\mathrm{MoS}_{2}$ : a new direct-gap semiconductor. Phys Rev Lett, 2010, 105: 136805

6 Tang Q, Zhou Z, Chen Z. Innovation and discovery of graphenelike materials via density-functional theory computations. Comput Mol Sci, 2015, 5: 360-379

7 Tang Q, Zhou Z. Graphene-analogous low-dimensional materials. Prog Mater Sci, 2013, 58: 1244-1315

8 Zhang SL, Hu YH, Hu ZY, et al. Hydrogenated arsenenes as planar magnet and Dirac material. Appl Phys Lett, 2015, 107: 022102

9 Antunez PD, Buckley JJ, Brutchey RL. Tin and germanium monochalcogenide IV-VI semiconductor nanocrystals for use in solar cells. Nanoscale, 2011, 3: 2399-2411

$10 \mathrm{Li} \mathrm{L}$, Chen Z, Hu Y, et al. Single-layer single-crystalline SnSe nanosheets. J Am Chem Soc, 2013, 135: 1213-1216

11 Mukherjee B, Cai Y, Tan HR, et al. NIR Schottky photodetectors based on individual single-crystalline GeSe nanosheet. ACS Appl Mater Interfaces, 2013, 5: 9594-9604
12 Vaughn Il DD, Patel RJ, Hickner MA, et al. Single-crystal colloidal nanosheets of GeS and GeSe. J Am Chem Soc, 2010, 132: 15170-15172

13 Xue DJ, Tan J, Hu JS, et al. Anisotropic photoresponse properties of single micrometer-sized GeSe nanosheet. Adv Mater, 2012, 24 : 4528-4533

14 Yoon SM, Song HJ, Choi HC. p-Type semiconducting GeSe combs by a vaporization-condensation-recrystallization (VCR) process. Adv Mater, 2010, 22: 2164-2167

15 Zhao LD, Lo SH, Zhang Y, et al. Ultralow thermal conductivity and high thermoelectric figure of merit in SnSe crystals. Nature, 2014, 508: 373-377

16 Hsueh H, Vass H, Clark S, et al. High-pressure effects in the layered semiconductor germanium selenide. Phys Rev B, 1995, 51: 16750

17 Makinistian L, Albanesi E. Ab initio calculations of the electronic and optical properties of germanium selenide. J Phys Cond Matt, 2007, 19: 186211

18 Rathor A, Sharma V, Heda N, et al. Compton profiles and band structure calculations of IV-VI layered compounds GeS and GeSe. Radiat Phys Chem, 2008, 77: 391-400

19 Yu LM, Degiovanni A, Thiry P, et al. Infrared optical constants of orthorhombic IV-VI lamellar semiconductors refined by a combined study using optical and electronic spectroscopies. Phys Rev B, 1993, 47: 16222

20 Kamal C, Ezawa M. Arsenene: two-dimensional buckled and puckered honeycomb arsenic systems. Phys Rev B, 2015, 91: 085423

21 Wu M, Fu H, Zhou L, et al. Nine new phosphorene polymorphs with non-honeycomb structures: a much extended family. Nano Lett, 2015, 15: 3557-3562

22 Guan J, Zhu Z, Tománek D. Phase coexistence and metal-insulator transition in few-layer phosphorene: a computational study. Phys Rev Lett, 2014, 113: 046804

23 Zhu Z, Tománek D. Semiconducting layered blue phosphorus: a computational study. Phys Rev Lett, 2014, 112: 176802

24 Guan J, Zhu Z, Tománek D. Tiling phosphorene. ACS nano, 2014, 8: $12763-12768$

25 Zhu Z, Guan J, Liu D, et al. Designing isoelectronic counterparts to layered group V semiconductors. ACS nano, 2015, 9: 8284-8290

26 Singh AK, Hennig RG. Computational prediction of two-dimensional group-IV mono-chalcogenides. Appl Phys Lett, 2014, 105: 042103

27 Segall M, Lindan PJ, Probert MA, et al. First-principles simulation: ideas, illustrations and the CASTEP code. J Phys Cond Matt, 2002, 14: 2717

28 Perdew JP, Burke K, Ernzerhof M. Generalized gradient approximation made simple. Phys Rev Lett, 1996, 77: 3865

29 White J, Bird D. Implementation of gradient-corrected exchange-correlation potentials in Car-Parrinello total-energy calculations. Phys Rev B, 1994, 50: 4954

30 Fischer TH, Almlof J. General methods for geometry and wave function optimization. J Phys Chem, 1992, 96: 9768-9774

31 Monkhorst HJ, Pack JD. Special points for Brillouin-zone integrations. Phys Rev B, 1976, 13: 5188

32 Heyd J, Scuseria GE, Ernzerhof M. Hybrid functionals based on a screened Coulomb potential. J Chem Phys, 2003, 118: 8207-8215

33 Gomes LC, Carvalho A. Phosphorene analogues: isoelectronic two-dimensional group-IV monochalcogenides with orthorhombic structure. Phys Rev B, 2015, 92: 085406

34 Zhang SL, Yan Z, Li YF, et al. Atomically thin arsenene and antimonene: semimetal-semiconductor and indirect-direct band-gap transitions. Angew Chem Int Ed, 2015, 54: 3112-3115

35 Wang X, Maeda K, Thomas A, et al. A metal-free polymeric photocatalyst for hydrogen production from water under visible light. Nat 
Mater, 2009, 8: 76-80

36 Scalise E, Houssa M, Pourtois G, et al. Vibrational properties of silicene and germanene. Nano Res, 2013, 6: 19-28

37 Lee C, Yan H, Brus LE, et al. Anomalous lattice vibrations of single-and few-layer $\mathrm{MoS}_{2}$. ACS nano, 2010, 4: 2695-2700

Acknowledgments This work was supported by the National Basic Research Program of China (2014CB931700), the National Natural Science Foundation of China (61222403 and 21403109) the Natural Science Foundation of Jiangsu province (BK20140769), and the Priority Academic Program Development of Jiangsu Higher Education Institutions (PAPD). We also acknowledge the Computer Network
Information Center (Supercomputing center) of Chinese Academy of Sciences (CAS) for the allocation of computing resource.

Author contributions Zhang S and Zeng H conceived the study, Liu S, Huang S, Cai B and Xie M performed the DFT calculations. Zhang S, Qu L, Zou Y, Hu Z and Yu X wrote the manuscript. All authors read and approved the final manuscript.

Conflict of interest The authors declare that they have no conflict of interest.

Supplementary information The supporting data are available in the online version of the paper.

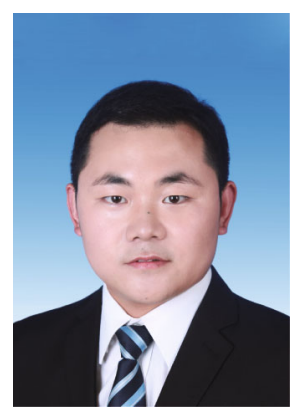

Shengli Zhang received his PhD degree from Beijing University of Chemical Technology in 2013. He then joined the Institute of Optoelectronics \& Nanomaterials, Nanjing University of Science and Technology. His research interests are focused on electronic or optoelectronic devices and applications based on atomic layer thick 2D materials.

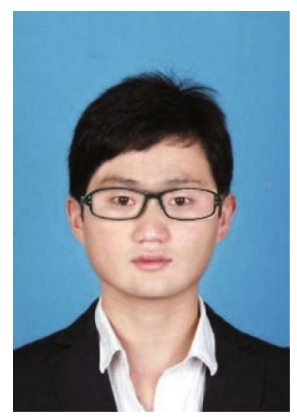

Ziyu Hu obtained his BSc degree and PhD degree from Beijing University of Chemical Technology. Then he worked as a postdoctoral fellow at Beijing Computational Science Research Center. He currently works at Algorithms Division of Beijing Computational Science Research Center, China. His research concerns designing solar energy materials for photoelectrocatalysis and topological property.

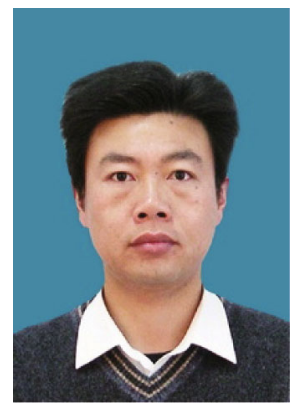

Haibo Zeng received his PhD degree from the Institute of Solid State Physics, Chinese Academy of Sciences in 2006. He later worked with Prof. Claus Klingshirn in 2007 at the University of Karlsruhe, Germany. In 2008, he joined Prof. Yoshio Bando's group at the National Institute for Materials Science (NIMS), Japan, under the support of Japan Society for the Promotion of Science (JSPS). In 2011, he returned to Nanjing University of Aeronautics and Astronautics as a full professor of materials science, and then moved to Nanjing University of Science and Technology in 2013 as a distinguished professor and director of the Institute of Optoelectronics \& Nanomaterials. His current research interest is low-dimensional semiconductor optoelectronics, including 2D materials (calculation and experiment) and QDs (synthesis, optics, photodetectors and LEDs). So far, he has published over 150 peer-review papers with citation more than 6,000 times.

中文摘要 本文利用密度泛函理论, 系统研究了五种单层 $\mathrm{GeSe}$ 晶型的结构、稳定性和电子结构特性, 并着重分析了其结构和电子性质 差异. 研究结果表明, 五种单层GeSe晶型均表现出稳定的半导体特性. 不同的是 $\beta$-GeSe、 $\gamma$-GeSe、 $\delta$-GeSe和 $\varepsilon-G e S e$ 晶型结构是间接带 隙半导体材料, 而 $\alpha-G e S e$ 是直接带隙半导体. 计算进一步提供了五种晶型结构的拉曼光谱和扫描隧道显微镜图像. 带边排布分析表明 $\beta-G e S e$ 单层材料适用于光催化分解水. 